\title{
Analytical model of oil pipeline overground transitions, laid in mountain areas
}

\author{
Andriy S. Velychkovych, Andriy V. Andrusyak, Tetiana O. Pryhorovska*, and Liubomyr Y. Ropyak \\ Ivano-Frankivsk National Technical University of Oil and Gas, 15 Karpatska Str., 76019, Ivano-Frankivsk, Ukraine
}

Received: 30 January 2019 / Accepted: 20 June 2019

\begin{abstract}
Abnormal climate changes cause adverse physical and geographical processes (erosion of soils, waterlogging, flooding, etc.) over the world. This situation stipulates study on effect of soil foundation malleability on pipeline transition strength because of its important diagnostic value.

The article develops an engineering approach for stress-strain state estimation of oil pipeline overground transitions, laid in mountainous areas. A mathematical statement was made and analytical solutions of the boundary-value problem were described, which take into account overland transition contact with soil foundation. In order to carry out the force analysis, a tubular rod simulated the oil pipeline contacting with the soil foundation on the adjacent to the overground transition section according to the Fuss-Winkler hypothesis. The overground transition was schematised with a thin-walled shell at the final stage of the assessment of strength. The maximum axial stresses were determined for overground transition operation, considering transition's two-dimensional thermoelastic state. The final assessment of the strength was carried out according to the energy criterion. The proposed method for the overground transition simulation makes possible considering the soil foundation property effects on the behavior of the oil pipeline transition and presents the results in the form of concise analytical expressions convenient for engineering practice. The conducted researches showed the effect of significant redistribution of loading in the oil pipeline overground transition caused by changes of soil foundation stiffness. Also, the boundary effect of perturbation of the stressed state in adjacent underground sections was found. It was determined the zone sizes the boundary effect has significant manifestations. The boundary state of the pipeline is most often achieved in its lower fibers at a short distance from the edge of the underground section. The obtained results of researches were tested on real constructions of overground transitions of oil pipeline laid in mountainous areas.
\end{abstract}

\section{Introduction}

Main oil pipelines usually pass a number of natural and artificial obstacles (ravines, mountain rivers, roads, technological developments, etc.) on mountain areas. These obstacles stipulate construction of transitions; and the beam transitions are the most widely used because of their cheapness in construction and easy operation $[1,2]$.

At the same time, the transitions of main pipelines are high-loaded constructions, because they are designed with lower strength factors than strength factors for other industries in order to decrease material usage [3]. Therefore, all essential factors that influence on the behavior of the oil pipeline during its operation should be considered

\footnotetext{
* Corresponding author: pryhorovska@gmail.com
}

within pipeline designing or calculating of strength and stiffness [4].

Long length of the oil pipeline stipulates the necessity to use the relevant method of stress-strain state calculation, because the pipeline wall thickness increasing at least $1 \mathrm{~mm}$ will cause a significant over-consumption of the material and financing.

A characteristic feature of the overground pipeline transition is its interaction with adjacent underground sections. Typically, the overground beam transitions of main pipelines are not rigidly fixed, but are ground-based (bottom trench) [5]. Soil is not only a loading factor for the transition, but also an environment of deformation. As a result, the task of pipeline stress-strain state definition is turned out to the task of the "pipeline - soils" system calculation.

This way, development of oil pipeline overground transition stress-strain state assessing methods is an actual practical task, the solution of which will contribute to mountainous pipeline safety increasing. 


\section{Literature review: Purpose and objectives of the research}

An analysis of modern research and publications indicates that there are some main approaches to oil pipeline overground transition stress-strain state definition and transition strength engineering assessment.

The first approach is schematization of real objects and development of their simplified mechanical and mathematical models by classical means of mechanics of a deformable solid. Usually, a both-sides rigidly fixed beam simulates an overground transition [2, 5-8]. This model is named a "rigid model" in mechanics.

Apparently, this approach greatly simplifies the problem, but at the same time leads to significant inaccuracies in the calculation, because this "rigid model" does not in any way take into account the soil foundation property effect on the overground transition strength. In particular, the "rigid model" usage for design calculations leads to over-utilization of material for transition, and therefore to unnecessary financial costs. Some authors do not develop a rod model, but use only the Barlow's formula with additional empirical coefficients $[1,8]$.

The second approach of research is usage of numerical simulations for stresses and deformations of the underground and overground sections of oil pipelines [9-13]. More often, researchers use the finite element method and universal software packages for automated engineering calculations as ANSYS, SolidWorks, Nastran, or low scope task applications, such as 3D PipeMaster. This way, soil is simulated by linear or nonlinear finite constraints. Constraints are installed in every node of the pipeline scheme. The main problem of such constraint using is the definition of soil resistance functions [12]. Besides, software packages provide non-linear models of solid medium for soil foundation in order to consider the consolidation and soil shaping processes [13]. In particular, the modern numerical methods for formulation and solving of elastic body and rock interaction contact problems are presented in $[14,15]$.

Another research approach provides application and development of classical methods of contact interaction mechanics to evaluate the stress state of tubular or plate elements, contacting with elastic bodies. In particular, the stress state and boundary equilibrium of pipelines constructed in the zone of active faults were investigated in the papers $[16,17]$. It is worth mentioning the class of problems related to contact interaction of a cut or crack section in thin-walled tubes [18-20], the study of cut and crackshaped defect creeping efficiency [21,22], and also stress state of pipes with flexible coatings [23]. This way, means of the contact mechanics make possible to present the pipeline-soil interaction as a model of layered body under distributed or local loadings. Estimated calculations for the strength and stiffness of layered structures for axially symmetric loads are presented in the papers [24, 25], and in the works of the local arbitrarily oriented loading [26, 27]. Approaches to the study of contact interaction of a cylindrical shell with an elastic body by simulations and laboratory experiments are given in $[28,29]$.
The authors put forward two main tasks in the presented herein study:

1. To develop a mechano-mathematical model of the oil pipeline overground transition in order to correctly consider transition's soil base property influence on the transition's strength.

2. To obtain concise analytical results that would be convenient for practical engineering calculations based on the developed model.

This way, the purpose of this work is the engineering methodology development for oil pipeline overground transition strength estimation.

The author's approach to calculation will make possible consideration of the soil foundation property effect on the transition's strength in order to obtain simple analytical results for design or diagnostic calculations.

\section{Method of analytical solutions}

\subsection{Problem statement}

Let's consider the scheme of one-run overground transition of the pipeline (Fig. 1a). The transition consists of the overground section $B C$ lengthed $2 l$ and adjacent underground sections $A B$ and $C K$, placed in the soil. The length of the underground sections is much larger than the overground one. Considering the identify of soil properties for underground sections and the fact that overground section is deformed symmetrically with respect to its center, it is enough to define the stress-strain state of the oil pipeline for the half of the $B C$ section and the $C K$ section.

Let's introduce the local systems of Cartesian coordinates (Fig. 1a), their origins refer to the middle point of the overground section $B C$ and the left edge of the underground section $C K$ respectively. A semi-continuous tubular rod with elastic base (Fig. 1b) simulates the stress-strain state of the pipeline section $C K$, which is placed in the soil. There was used the Fuss-Winkler elastic foundation model. For this foundation, the distributed reaction at each point of the pipe is proportional to the deflection of the oil pipeline and depends on the properties of the soil. The overground section $B C$ of the oil pipeline is simulated by a straight lateral bend of the tubular rod of finite length. Own weight of the oil pipeline, oil weight in the pipes, soil weight to the underground section are simulated by the uniform distributed loads with intensities $q_{1}$ and $q_{2}$. Additionally, we have considered the stresses caused by the internal pressure and temperature difference, which are constant over the length. It should be mentioning, that the pipeline will be simulated as a thin-walled shell after its force and deformation analysis and more detailed consideration of the pipeline's strength. All researches are provided in geometrically and physically formulation.

The system of differential equations of equilibrium in terms of displacement components, which define deformation of the oil pipeline sections, is presented below: 


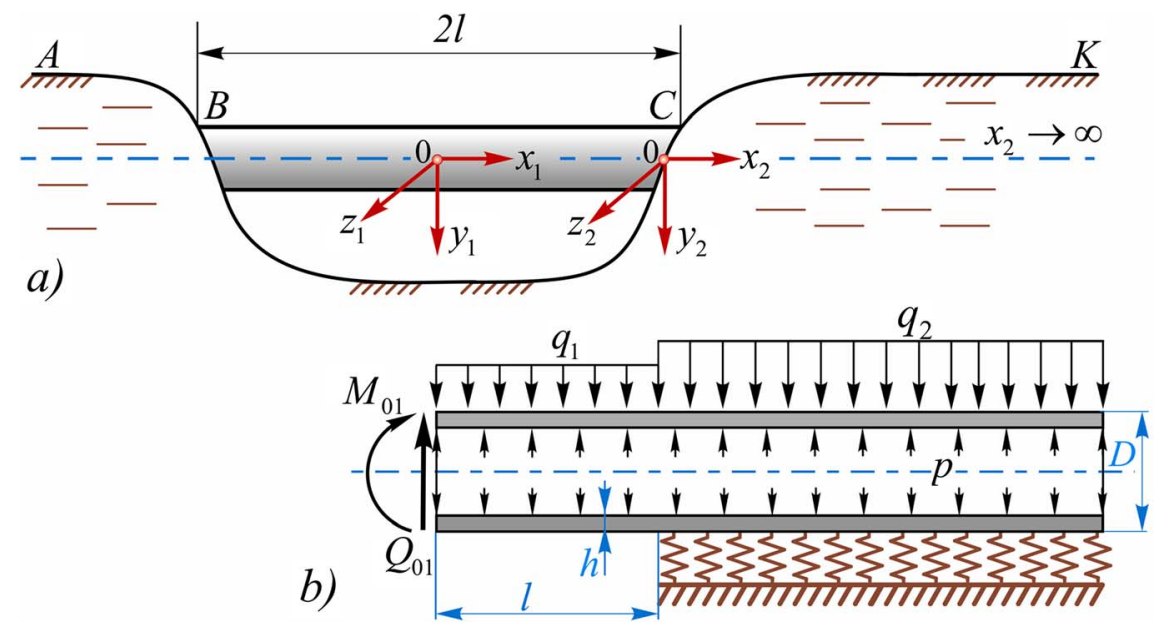

Fig. 1. Scheme of the overground transition of the oil pipeline: (a) general view of the object of research; (b) calculation model.

$$
\begin{gathered}
E J_{z} \frac{\mathrm{d}^{4} w_{y 1}}{\mathrm{~d} x_{1}^{4}}+q_{1}=0, \quad x_{1} \in[0, l) ; \\
E J_{z} \frac{d^{4} w_{y 2}}{d x_{2}^{4}}+D k_{y} w_{y 2}=q_{2}, \quad x_{2} \in[0, \infty),
\end{gathered}
$$

where $w_{y 1}, w_{y 2}$ are transverse displacement of the overground and underground sections of the oil pipeline respectively; $E$ is Young's module of pipe material; $J_{z}$ is the axial moment of inertia of the pipe cross section; $D$ is diameter of pipes; $k_{y}$ is coefficient of bedding of soil foundation.

\subsection{Solution of the boundary value problem}

Fourfold sequential integration of equation (1) made us possible obtaining a deflection function and its derivatives. The general equation of the oil pipeline internal loadings is as follows:

$$
\left\{\begin{array}{c}
E J_{z} \frac{\mathrm{d}^{3} w_{y 1}}{\mathrm{~d} x_{1}^{3}}=Q_{y 1}\left(x_{1}\right)=Q_{01}-q_{1} x_{1} \\
E J_{z} \frac{\mathrm{d}^{2} w_{y 1}}{\mathrm{~d} x_{1}^{2}}=M_{z 1}\left(x_{1}\right)=M_{01}+Q_{01} x_{1}-\frac{1}{2} q_{1} x_{1}^{2} .
\end{array}\right.
$$

Displacement equations:

$$
\left\{\begin{array}{c}
\frac{\mathrm{d} w_{y 1}}{\mathrm{~d} x_{1}}=\varphi_{1}\left(x_{1}\right)=\varphi_{01}+\frac{M_{01} x_{1}}{E J_{z}}+\frac{Q_{01} x_{1}^{2}}{2 E J_{z}}-\frac{q_{1} x_{1}^{3}}{6 E J_{z}} \\
w_{y 1}\left(x_{1}\right)=w_{01}+\varphi_{01} x_{1}+\frac{M_{01} x_{1}^{2}}{2 E J_{z}}+\frac{Q_{01} x_{1}^{3}}{6 E J_{z}}-\frac{q_{1} x_{1}^{4}}{24 E J_{z}} .
\end{array}\right.
$$

where $Q_{y 1}\left(x_{1}\right), M_{z 1}\left(x_{1}\right), \varphi_{1}\left(x_{1}\right)$ are functions of transverse forces, bending moments and angles of rotation in the overground section of the oil pipeline; $Q_{01}, M_{01}$ and $\varphi_{01}, w_{01}$ are static and geometric initial parameters.

The next stage is the development of the general solution of equation (2).

Assuming $E J_{z}=$ const for the oil pipe, equation (2) is presented as follow:

$$
\frac{\mathrm{d}^{4} w_{y 2}}{\mathrm{~d} x_{2}^{4}}+4 \beta^{4} w_{y 2}=\frac{q_{2}}{E J_{z}}, x_{2} \in[0, \infty),
$$

where the introduced designation $\beta=\sqrt[4]{k_{y} D / 4 E J_{z}}$ is a coefficient which depends on the soil foundation stiffness and pipeline bend stiffness. follows:

The general integral of equation (5) is represented as

$$
\begin{gathered}
w_{y 2}\left(x_{2}\right)=e^{-\beta x_{2}}\left(C_{1} \sin \beta x_{2}+C_{2} \cos \beta x_{2}\right)+ \\
+e^{\beta x_{2}}\left(C_{3} \sin \beta x_{2}+C_{4} \cos \beta x_{2}\right)+\frac{q_{2}}{D k_{y}}
\end{gathered}
$$

where the constants of integration $C_{1}-C_{4}$ are determined by the boundary conditions at the edges of the underground area.

The follow boundary conditions were defined to define the constants of integration. The first two of them refer to the fact, that the transverse force and bending moment on the right edge of the underground section of the oil pipeline $C K$ are zero:

$$
\left.E J_{z} \frac{\mathrm{d}^{3} w_{y 2}}{\mathrm{~d} x_{2}^{3}}\right|_{x_{2} \rightarrow \infty}=0,\left.\quad E J_{z} \frac{\mathrm{d}^{2} w_{y 2}}{\mathrm{~d} x_{2}^{2}}\right|_{x_{2} \rightarrow \infty}=0 .
$$

Then, considering the conditions (7) of the expression (6) we obtain: $C_{3}=C_{4}=0$.

The following two conditions refer to the fact that the second and third derivatives of the transverse displacement function are equal to the static initial parameters of the $C K$ section:

$$
\left.E J_{z} \frac{\mathrm{d}^{2} w_{y 2}}{\mathrm{~d} x_{2}^{2}}\right|_{x_{2} \rightarrow 0}=M_{02},\left.E J_{z} \frac{\mathrm{d}^{3} w_{y 2}}{\mathrm{~d} x_{2}^{3}}\right|_{x_{2} \rightarrow 0}=Q_{02} .
$$

This form of boundary conditions is a methodical step to present the last relations in a simple compact form. Meeting the conditions (8):

$$
C_{1}=-\frac{M_{02}}{2 \beta^{2} E J_{z}}, \quad C_{2}=\frac{\beta M_{02}+Q_{02}}{2 \beta^{3} E J_{z}} .
$$


The complete solution of equation (5) for the underground section in the form of formulas for determining transverse forces $Q_{y^{2}}\left(x_{2}\right)$, bending moments $M_{z 2}\left(x_{2}\right)$, angles of rotation $\varphi_{2}\left(x_{2}\right)$ and transverse displacements $w_{y_{2}}\left(x_{2}\right)$ was obtained by substituting the integration constants (9) to the (6):

$$
\begin{gathered}
Q_{y 2}\left(x_{2}\right)=-2 \beta M_{02} \eta_{4}\left(x_{2}\right)+Q_{02} \eta_{2}\left(x_{2}\right), \\
M_{z 2}\left(x_{2}\right)=M_{02} \eta_{1}\left(x_{2}\right)+\frac{Q_{02}}{\beta} \eta_{4}\left(x_{2}\right) ; \\
\varphi_{2}\left(x_{2}\right)=\frac{2 \beta^{2}}{D k_{y}}\left[2 \beta M_{02} \eta_{3}\left(x_{2}\right)+Q_{02} \eta_{1}\left(x_{2}\right)\right], \\
w_{y_{2}}\left(x_{2}\right)=\frac{2 \beta}{D k_{y}}\left[\beta M_{02} \eta_{2}\left(x_{2}\right)+Q_{02} \eta_{3}\left(x_{2}\right)\right]+\frac{q_{2}}{D k_{y}} .
\end{gathered}
$$

The new definitions are introduced in formulas (10) and (11):

$$
\begin{gathered}
\eta_{1}\left(x_{2}\right)=\mathrm{e}^{-\beta x_{2}}\left(\cos \beta x_{2}+\sin \beta x_{2}\right) ; \\
\eta_{2}\left(x_{2}\right)=\mathrm{e}^{-\beta \mathrm{x}_{2}}\left(\cos \beta x_{2}-\sin \beta x_{2}\right) ; \\
\eta_{3}\left(x_{2}\right)=\mathrm{e}^{-\beta x_{2}} \cos \beta x_{2} ; \quad \eta_{4}\left(x_{2}\right)=\mathrm{e}^{-\beta x_{2}} \sin \beta x_{2} .
\end{gathered}
$$

It remains to specify the conditions at the beginning of the overground section of the oil pipeline and the conditions of compatibility and continuity at the connection point of the overground $B C$ and the underground $C K$ sections. Consequently, if $x_{1}=0$, then the conditions of the structure symmetry stipulate that the tangent line to the deflection line is parallel to the axis, so that $\varphi_{01}=0$ and $Q_{01}=0$. The system of conditions for the connection point of the sections is as follows:

$$
\begin{gathered}
Q_{y 1}(l)=Q_{y 2}(0), M_{z 1}(l)=M_{z 2}(0) ; \\
\varphi_{y 1}(l)=\varphi_{y 2}(0), w_{y 1}(l)=w_{y 2}(0) .
\end{gathered}
$$

The relations to define the initial parameters were obtained by meeting the conditions (12), from the relations (3), (4) and (10), (11):

$$
\begin{gathered}
M_{01}=\frac{q_{1} l}{\frac{l}{E J_{z}}+2 \zeta \beta}\left[\frac{l^{2}}{6 E J_{z}}+\zeta(1+\beta l)\right] \\
M_{02}=-M_{01}+\frac{1}{2} q_{1} l^{2}, Q_{02}=q_{1} l ; \\
w_{01}=-\zeta\left(M_{02}+\frac{Q_{02}}{\beta}\right)-\frac{1}{E J_{z}}\left(\frac{M_{01} l^{2}}{2}-\frac{q_{1} l^{4}}{24}\right)+\frac{q_{2}}{D k_{y}},
\end{gathered}
$$

where $\zeta=2 \beta^{2} / D k_{y}$.

The obtained solutions (3), (4) and (10), (11) with initial parameters make possible to carry out a full analysis of the distribution of forces and deformations in the overground pipeline transition, taking into account soil property influence on the structure's behavior.

\section{Numerical analysis of the results}

\subsection{Force analysis}

The follow real problem was specified as a sample in order to depict its solution and the obtained results. Figure 2 shows a general view of the overground transition of a pipeline that is laid out in mountain areas. For example, we chose a one-run transition (in Fig. 2 it is depicted on the left).

The length of the overground run of the transition is $25 \mathrm{~m}$, the soil foundation is loam with crushed stone with the bed coefficient of $k_{y}=10 \mathrm{MN} / \mathrm{m}^{3}$, the soil specific gravity is $0.027 \mathrm{~N} / \mathrm{sm}^{3}$. The material of the pipes is the steel with the following characteristics: Young's modulus $2.06 \times 10^{5} \mathrm{MPa}$, Poisson's coefficient -0.3 , linear expansion coefficient - $12 \times 10^{-6} \mathrm{~K}^{-1}$, yield strength - $364 \mathrm{MPa}$, pipe diameter $720 \mathrm{~mm}-$, thickness of the pipe wall $-9.5 \mathrm{~mm}$.

The temperature of the air during the installation of the transition was $22{ }^{\circ} \mathrm{C}$. The pipeline transports crude oil, the working pressure is $-3.1 \mathrm{MPa}$.

The force distribution for the proposed model was calculated according to the standard methods [7, 30, 31]. The forces were as follows: $q_{1}=5.2 \mathrm{kN} / \mathrm{m}$ and $q_{2}=28.5 \mathrm{kN} /$ $\mathrm{m}$ for the oil pipeline filled with the product and $q_{1}=1.7 \mathrm{kN} / \mathrm{m}$ and $q_{2}=25 \mathrm{kN} / \mathrm{m}$ for the empty pipeline.

Figures 3 and 4 show internal load distribution along the main characteristic cross-sections of the oil pipeline (we present the plots in a stationary system of the Cartesian coordinates, the origin of this system is at the middle point of the overground section). These plots indirectly describe the stress state of the construction. The maximal transverse force $65 \mathrm{kN}$ is observed at the connection of the overground and underground sections, transverse force is zero in the middle of the overground section.

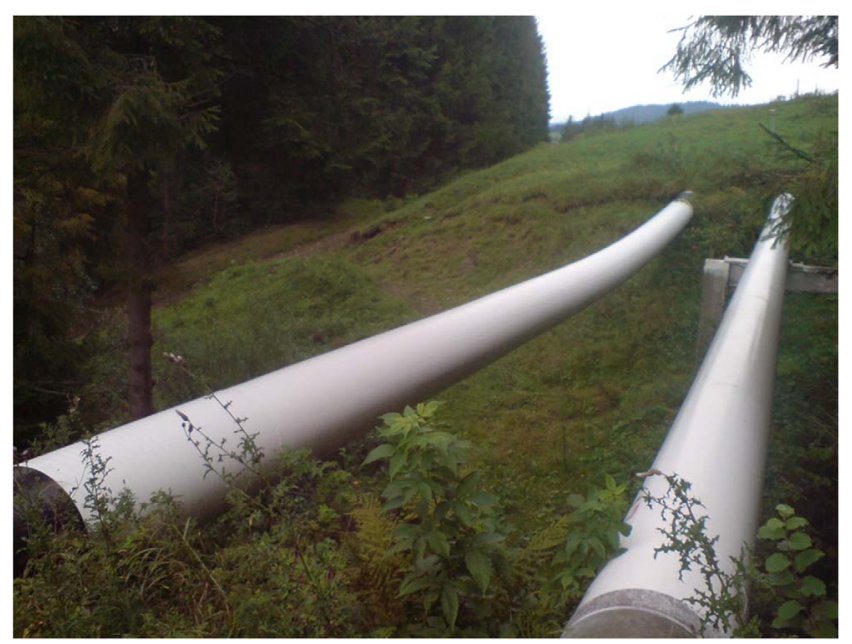

Fig. 2. The overground transition of the "Druzhba" oil pipeline (Carpathian mountains, Ukraine). 


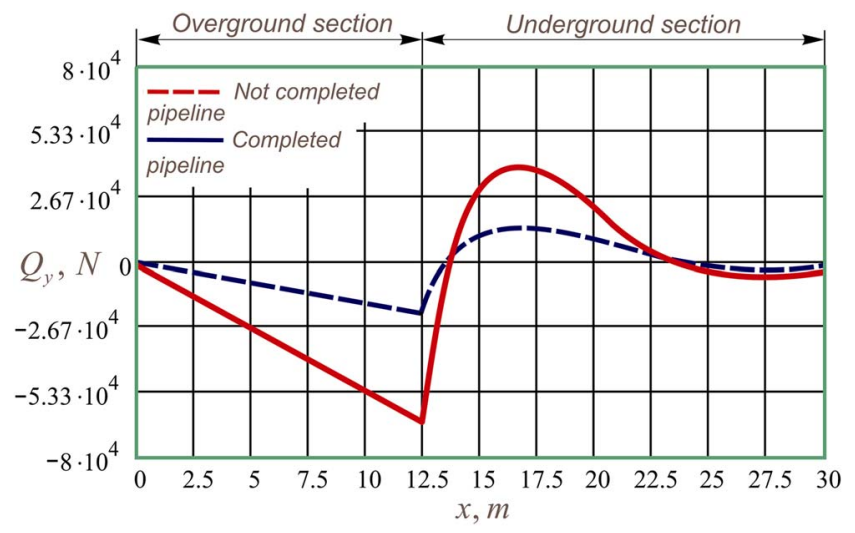

Fig. 3. Distribution of transverse forces in the oil pipeline.

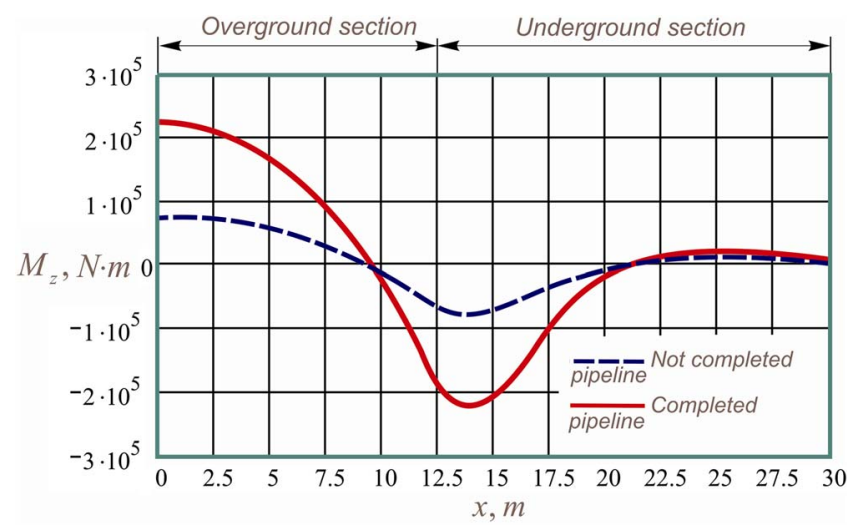

Fig. 4. Distribution of bending moments in the oil pipeline.

Another significant transverse force peak of $58 \mathrm{kN}$ is observed in the underground area at a distance of $3 \mathrm{~m}$ from the edge of the section. Apparently, that it is not desirable to place welded or any other connections of the oil pipeline at the cross-sections with peak values of transverse forces. Besides, there are two dangerous cross-sections in refer to the bending moment distribution. The bending moment of $220 \mathrm{kN} \mathrm{m}$ causes compressive normal stresses in the upper fibers of the pipes in the middle of the overground section.

The bending moment magnitude of $232 \mathrm{kN} \mathrm{m}$ is observed at a distance of $1.4 \mathrm{~m}$ from the edge of the underground area and causes compressive normal stresses in the lower fibers of the pipes. The maximum internal stresses for the pipeline filled by oil are, averagely, $67 \%$ higher than for the empty one.

The presented plots are characterized by the wave-like changes of the loading at the beginning of the underground section of the oil pipeline with gradual attenuation at the distance from the edge of this section. This effect can be named the boundary effect of changes in the stressed state of the underground section of the transition, and the length at which this effect is significantly manifested - the length of the boundary effect.
The attenuation of the effect is explained by the multiplier $\mathrm{e}^{-\beta x_{2}}$ in the functions $\eta_{\mathrm{i}}$ (10).

This multiplier goes to zero, if the multiplier $\beta x_{2}$ goes to infinity. We can estimate the length of the boundary effect for our task by using the function $\mathrm{f}\left(\beta x_{2}\right)=\mathrm{e}^{-\beta x_{2}}$.

If $x_{2}=0$, then this function is equal to one. If $\beta x_{2}=\pi$, then $\mathrm{f}(\pi)=0.046$ that is, with an accuracy of up to $5 \%$, it can be considered a function that is dampened. From equality, we calculate that for the underground section of the oil pipeline transition, the boundary effect will have significant manifestations at a length of $11.2 \mathrm{~m}$.

\subsection{Analysis of displacements}

Figures 5 and 6 show the distribution of displacements along the pipeline characteristic sections. The graphs describe the deformation of the considered part of the structure. The main extremum of the transverse displacement function is above the center of the overground transition. The derivative of this function is the angle of inclination of the axis of the pipe above the transition center and is equal to zero. The oil pipeline is slightly raised in the ground. The second local extremum of transverse displacement is observed at the distance of $7.5 \mathrm{~m}$ from the beginning of the underground section. In general, more detailed analysis of the deflections showed that, the function $w_{y}(x)$ may have one or three extremes depending on the stiffness of the soil foundation.

\subsection{Influence of soil foundation malleability}

Soil foundation properties for pipe laying differ depending on the terrain, the depth of the trench, and so on. We would like to note that resistance to the pipe movement of even one type of soil may vary considerably depending on the season or weather conditions [8]. Today, there are abnormal climate changes in a number of mountain regions (not characteristic for these territories), which provoke unfavorable physical and geographical processes (erosion of soils, waterlogging, flooding, etc.). In these cases, consideration of unexpected changes in the soil foundation malleability

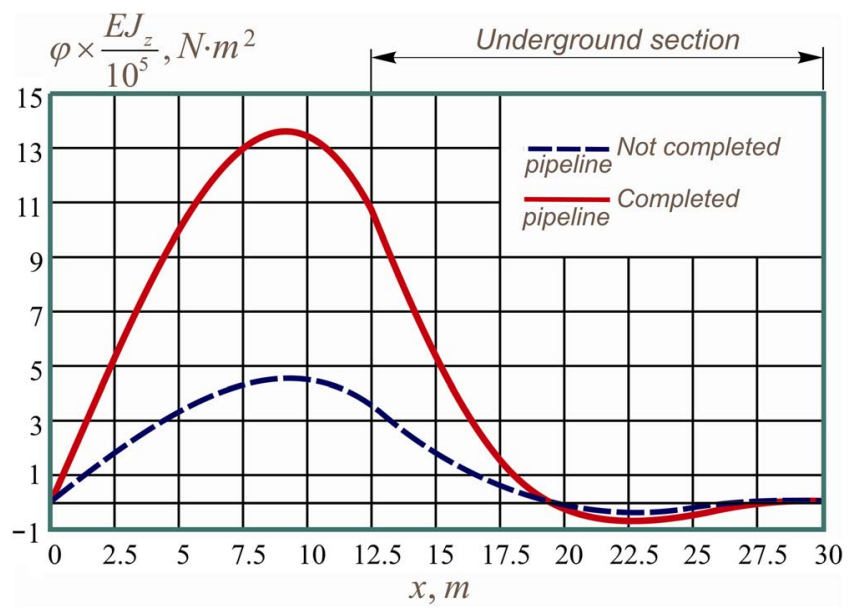

Fig. 5. Angles of rotation of oil pipeline sections. 


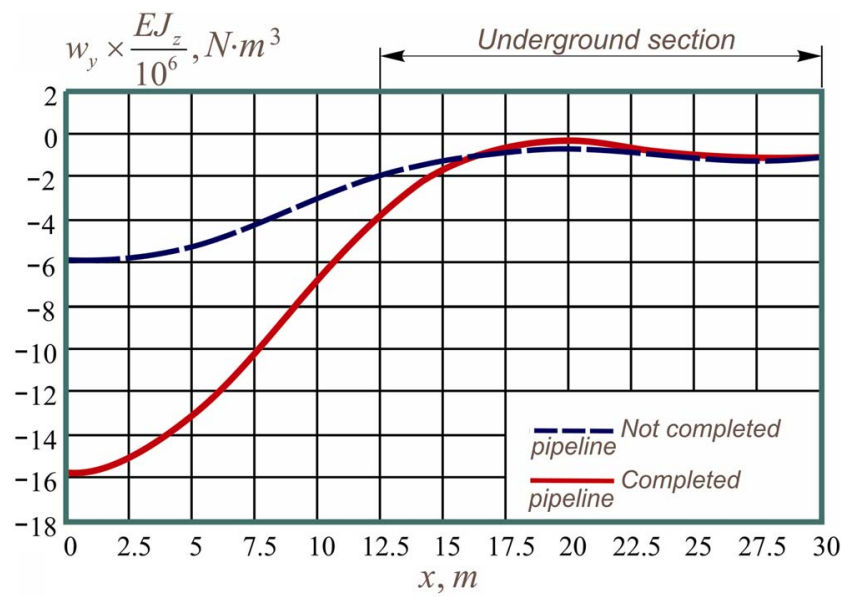

Fig. 6. Transverse displacement (deflection) of the oil pipeline.

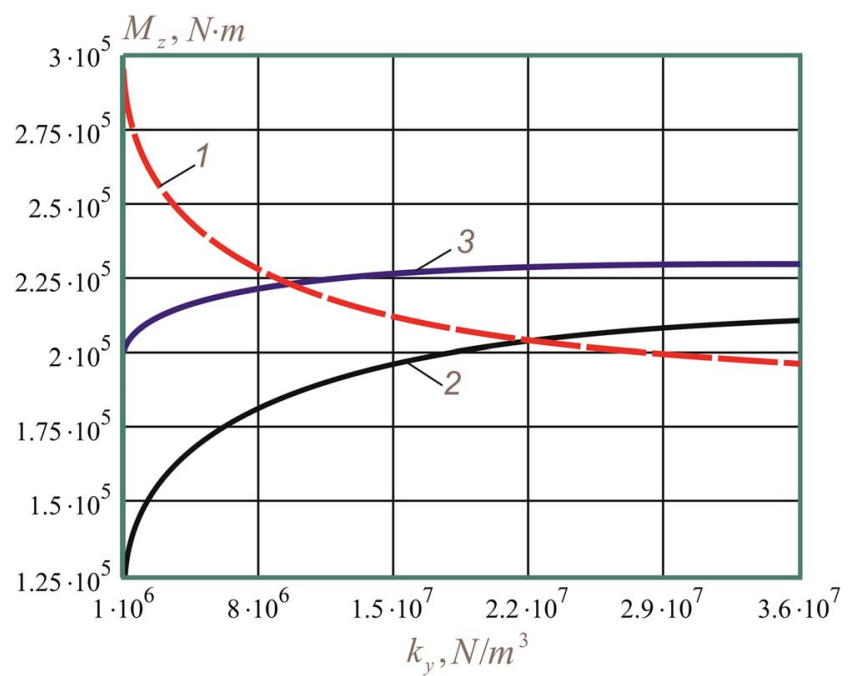

Fig. 7. Bending moments in the oil pipeline related the soil foundation properties: (1) bending moment in the middle point of the overground section; (2) bending moment at the edge of underground section; (3) maximum bending moment in the underground section.

and their influence on transition's strength has important diagnostic matter. Figure 7 shows the relations between the absolute values of bending moments in the most loaded transverse cross-sections of the oil pipeline and the soil foundation bedding coefficient.

These relations show pipeline stiffness increasing refers to the decreasing of the bending moments in the characteristic sections of the underground sections. Increasing of the soil foundation stiffness is accompanied by decreasing the distance between the edge of the underground section and the cross-section with the maximum bending moment. A further increasing of the bed coefficient causes gradually approach of bending moments (curves 1 and 2) to values, which can be obtained by the elementary "hard" model of the overground transition.

\subsection{Analysis of the strength of the overground transition}

Analysis of the strength of the overground transition is the final stage its strength assessment. Taking into account the two-dimensional thermoelastic state of the oil pipeline, the axial tension in its outer fibers will be presented as follows:

$$
\sigma_{x}=\mu \sigma_{\theta} \pm \sigma_{T} \pm \sigma_{M_{z}},
$$

where $\sigma_{\theta}$ is circular stresses caused by internal pressure, $\sigma_{T}$ is temperature stresses, $\sigma_{M_{z}}$ is normal bending stresses, $\mu$ is Poisson coefficient.

Let's detail the last expression:

$$
\sigma_{x}=\mu \frac{p D}{2 h}-E \alpha_{t} \Delta t \pm 4 \frac{M_{z}(x)}{\pi D^{2} h},
$$

where $p$ is internal pressure in the oil pipeline, $\alpha_{t}$ is coefficient of linear expansion, $\Delta t$ is temperature difference.

We neglected the tangential stresses caused by transverse forces, because they are essentially less than the normal stresses caused by the bending moment.

The stressed state of the oil pipeline material is flat with the main stresses $\sigma_{x}$ and $\sigma_{\theta}$. We formulate the condition of strength of the oil pipeline as a thin-walled shell. We use the energy criterion of Guber-Mises, according to which the equivalent stresses will be determined by the relation:

$$
\sigma_{\mathrm{td}}=\sqrt{\sigma_{x}^{2}+\sigma_{\theta}^{2}-\sigma_{x} \sigma_{\theta}} .
$$

The following condition should be met to ensure the strength of the structure: $\sigma_{\mathrm{td}} \leq[\sigma]$, where $[\sigma]$ is the permissible stress for the material of the structure, taking into account the coefficients of the stock, working conditions, reliability, etc.

Figures 8 and 9 show the distribution of axial and equivalent stresses in the overground transition of the oil pipeline and the adjacent underground sections. These relations were developed for a case without temperature difference considering. Under these conditions of loading, the boundary condition of the oil pipeline is firstly achieved in the

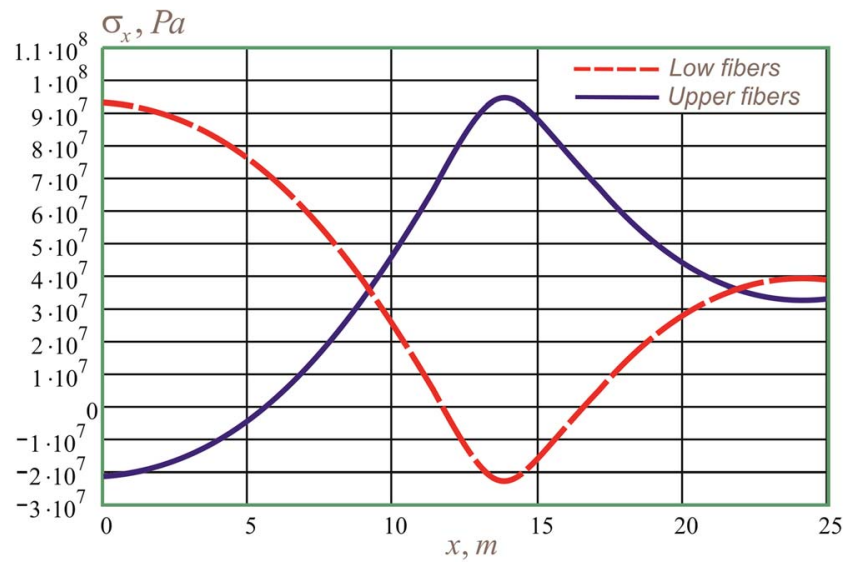

Fig. 8. Axial stresses in the upper and lower fibers of the overground transition of the oil pipeline. 


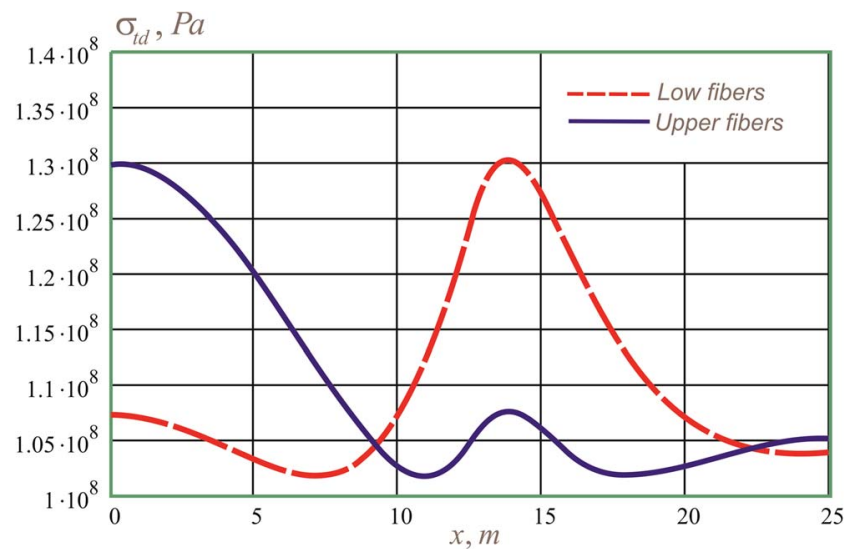

Fig. 9. Equivalent stresses in the upper and lower fibers of the overground transition of the oil pipeline.

lower compressed fibers at the distance of $1.4 \mathrm{~m}$ from the edge of the underground section.

\subsection{Analysis of the temperature stresses influence on strength}

The considered overground transition was mounted at the ambient temperature of $22^{\circ} \mathrm{C}$. The additional temperature stresses occur, if the initial temperature of the oil pipeline material increase by $\Delta t$ within pipeline transition operation. We assume the follow possible range of temperature changes: uniform heating by $20{ }^{\circ} \mathrm{C}$ or uniform cooling by

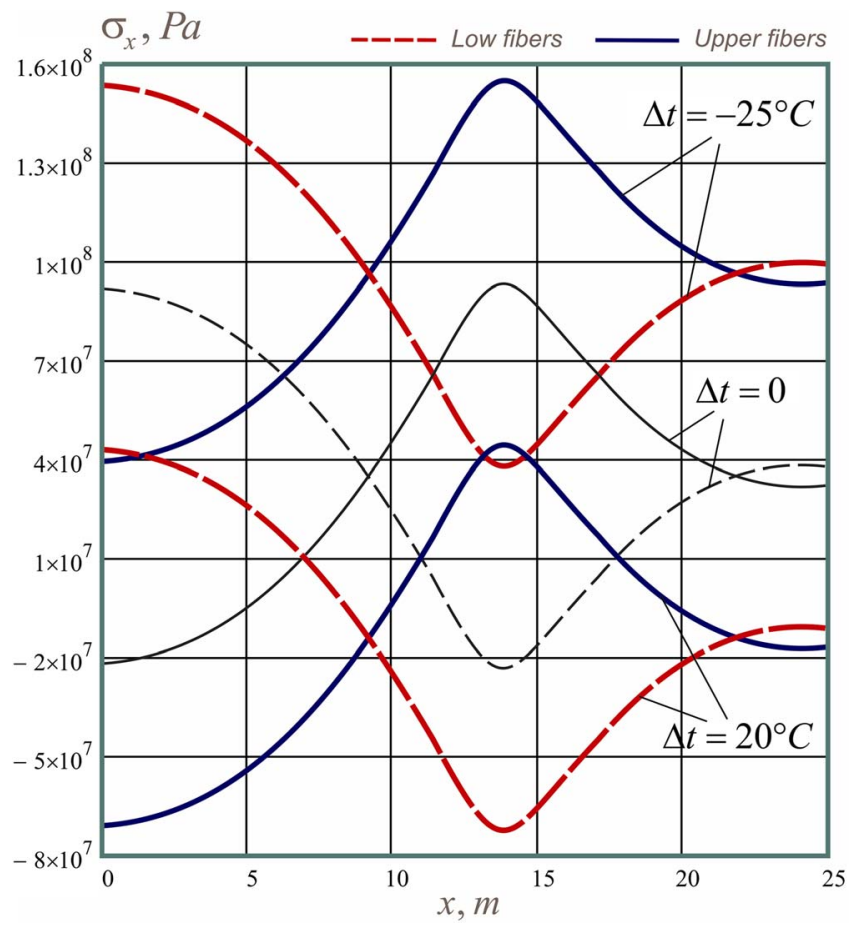

Fig. 10. Axial stresses in the overground transition of the oil pipeline, with consideration of temperature effects.

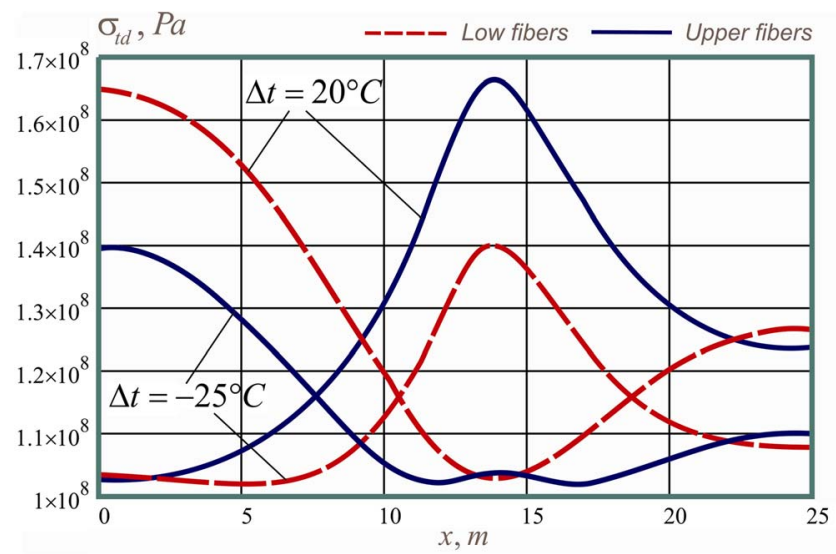

Fig. 11. Equivalent stresses with consideration of temperature effects.

$25^{\circ} \mathrm{C}$. This temperature range is typical for the climatic zone of the transition.

Figures 10 and 11 show the distribution of axial and equivalent stresses in the overground transition of the oil pipeline with consideration of possible temperature effects. Heating causes the additional compressive axial stresses and cooling causes the additional stretching effects in the material of the pipes.

This effect is indicated by the location of the axial stress graph lines (Fig. 10) if $\Delta t=20{ }^{\circ} \mathrm{C}$ and $\Delta t=-25{ }^{\circ} \mathrm{C}$ comparing with $\Delta t=0$. The heating of the overground transition makes the most significant effect on the magnitude of equivalent stresses concerning the final assessment of strength. In particular, heating by the $20{ }^{\circ} \mathrm{C}$ causes the maximum equivalent stresses increasing by $27 \%$.

\section{Conclusion}

The article develops an engineering approach for stressstrain state estimation of oil pipeline overground transitions, laid in mountainous areas. In order to carry out the force analysis, a tubular rod simulated the oil pipeline contacting with the soil foundation on the adjacent to the overground transition section according to the Fuss-Winkler hypothesis. A non-momentous shell presented the overground transition at the final stage of the strength assessment.

This approach, used at the structure simulation stage, makes possible to correctly consider the influence of the soil foundation property on transition's strength and thus obtain the results as simple analytical formulations for engineering practice.

The proposed methodological approaches were implemented for strength assessing of the real overground transition of the oil pipeline laid in mountain areas.

The attention is paid to the nature of loading redistribution in the oil pipeline with changes in the stiffness of the ground foundation and on the boundary effects that occur in adjacent areas of the overground transition. 
It was determined the maximum axial stresses with consideration of the two-dimensional thermoelastic state of the oil pipeline during its operation. The final strength estimate is based on the energy criterion.

It is determined that the properties of the soil foundations significantly affect the stress-strain state of the overground transition. In particular, increasing of the soil foundation stiffness is accompanied by bending moments gradually decreasing (and hence the stresses) in the overground section of the transition, and the maximum bending moment in the underground section increases. Obviously, covering of the trench indigenous rock with a layer of soil with the required bed coefficient can affect the strength of the overground transition.

The boundary effect of changes in the stressed state of the underground transition section and the length of this effect manifestation is determined. It is shown that the boundary state of the pipeline is first achieved in its lower fibers at a short distance from the edge of the underground area.

It was made an estimation temperature stress effect on the overground transition strength. It has been determined that the final value of equivalent stresses can essentially depend on temperature loads.

In general, the overground transition model, proposed by the authors, makes possible strict mathematical formulation of a number of practical problems and obtaining their solutions in an analytical or numerical-analytic form. To do this, it is enough consider the boundary conditions in the presented solutions, which correspond to the particular engineering task. For example, these calculations are the design calculations of ground transitions with intermediate supports or suspensions, the assessment of the strength of the pipelines in the mountain zones of slow slipping of the soil (when there is a cavity underneath the pipeline), the determination of stresses in underground pipelines constructed in karst zones, etc.

\section{References}

1 David P.A., Jr Hermanson, Wagner M.J. (2015) Challenges facing large-diameter pipelines crossing mountains, Pipeline Gas J. 242, 1.

2 Shashi Menon E. (2011) Pipeline planning and construction field manual, Gulf Professional Publishing, Lake Havasu City, AZ, USA.

3 Orynyak I.V., Lokhman I.V., Sidor M.D., Radchenko S.A. (2009) Analysis of the stress-strain state of an air crossing of pipeline in the course of repair, Strength Mater. 41, 581-591.

4 Timashev S.A., Bushinskaya A.V. (2016) Assessment of the reliability level embedded in pipeline design codes, $J$. Pipeline Eng. 15, 2, 121-131.

5 Oswell J.M. (2016) Soil mechanics for pipeline stress analysis, Naviq Consulting Inc., Calgary, Canada.

6 Adebanjo O., Simms N. (2016) Upheaval buckling of pipelines, J. Pipeline Eng. 15, 3, 157-168.

7 Antaki G.A. (2003) Piping and pipeline engineering: Design, construction, maintenance, integrity, and repair, Marcel Dekker Inc., New York, USA.

8 Borodavkin P.P. (2011) Underground trunk pipelines, Energy Press, Moscow, Russia, 479 p.
9 National Oilwell Varco (2013) Engineering \& piping design guide, Sand Springs, Oklahoma, USA.

10 Mark L.Talesnick, Frydman Sam (2018) Soil pressure and pipe deformation measurements for characterizing flexible pipe-soil systems under shallow cover, J. Pipeline Syst. Eng. Pract. 9, 1.

11 Bakhtyar F., Kenny S. (2014) Development of a fatigue life assessment tool for pipelines with local wrinkling through physical testing and numerical modelling, ASME. International Conference on Offshore Mechanics and Arctic Engineering 6B: Pipeline and Riser Technology, San Francisco, California, USA, June 8-13, 2014.

12 Yavarov A.V. (2012) Numerical simulation of the resistance of the soil massif to the subter-ranean pipeline displacement, Electr. Sci. J. Oil Gas Bus. 3, 360-374.

13 Elshimi T.M., Moore I.D. (2013) Modeling the effects of backfilling and soil compaction beside shallow buried pipes, J. Pipeline Syst. Eng. Pract. 4, 4.

14 Pryhorovska T.O. (2017) Study on rock reaction force depending on PDC cutter placement, Mach. Sci. Technol. 21, 1, 37-66.

15 Pryhorovska T.A., Chaplinskiy S.S. (2018) Finite element modeling of rock mass cutting by cutters for PDC drill bits, Neftyanoe Khozyaystvo - Oil Ind. 1, 38-41.

16 Shats'kyi I.P., Struk A.B. (2009) Stressed state of pipeline in zones of soil local fracture, Strength Mater. 41, 5, 548-553.

17 Zhang J., Liang Z., Han C.J. (2015) Finite element analysis of wrinkling of buried pressure pipeline under strike-slip fault, Mechanika 21, 3, 180-186.

18 Shats'kyi I.P., Makoviichuk M.V. (2009) Analysis of the limiting state of cylindrical shells with cracks with regard for the contact of crack lips, Strength Mater. 41, 5, 560-565.

19 Shatskii I.P., Makoviichuk N.V. (2011) Effect of closure of collinear cracks on the stress-strain state and the limiting equilibrium of bent shallow shells, J. Appl. Mech. Tech. Phys. 52, 3, 464-470.

20 Dovbnya K.M., Hryhorchuk Y.V (2016) Stressed state of shell of double curvature with two collinear cracks under bending, J. Math. Sci. 212, 1, 98-105.

21 Shats'kyi I.P. (2015) Limiting equilibrium of a plate with partially healed crack, Mater. Sci. 51, 3, 322-330.

22 Marukha V.I., Panasyuk V.V., Sylovanyuk V.P. (2014) Injection technologies for the repair of damaged concrete structures, Springer, New York, NY.

23 Shatskyi I., Popadyuk I., Velychkovych A. (2018) Hysteretic properties of shell dampers, in: J. Awrejcewicz (ed), Dynamical systems in applications. DSTA 2017, Springer proceedings in mathematics \& statistics. Springer, Cham, Switzerland.

24 Popadyuk I.Y, Shatskyi I.P., Shopa V.M., Velychkovych A. S. (2016) Frictional interaction of a cylindrical shell with deformable filler under nonmonotonic loading, J. Math. Sci. 215, 2, 243-253.

25 Velichkovich A.S., Dalyak T.M. (2015) Assessment of stressed state and performance characteristics of jacketed spring with a cut for drill shock absorber, Chem. Petrol. Eng. (New York: Springer) 51, 188-193.

26 Shatskyi I.P., Ropyak L.Y., Makoviichuk M.V. (2016) Strength optimization of a two-layer coating for the particular local loading conditions, Strength Mater. 48, 5, 726-730.

27 Ropyak L.Y., Shatskyi I.P., Makoviichuk M.V. (2017) Influence of the oxide-layer thickness on the ceramic-aluminium coating resistance to indentation, Metallofiz. Noveishie Tekhnol. 39, 4, 517-524. 
28 Velichkovich A., Dalyak T., Petryk I. (2018) Slotted shell resilient elements for drilling shock absorbers, Oil Gas Sci. Technol. - Rev. IFP Energies nouvelles 73, 34.

29 Velichkovich A.S., Popadyuk I.I., Shopa V.M. (2011) Experimental study of shell flexible component for drilling vibration damping devices, Chem. Petrol. Eng. (New York: Springer) 46, 518-524.
30 Panevnik D.A., Velichkovich A.S. (2017) Assessment of the stressed state of the casing of the above-bit hydroelevator, Neftyanoe Khozyaystvo - Oil Ind. 1, 70-73.

31 Vlasiy O., Mazurenko V., Ropyak L., Rogal O. (2017) Improving the aluminum drill pipes stability by optimizing the shape of protector thickening, EEJET 85, 25-31. 